\title{
Technology in Locomotion and Domotic Control for Quadriplegic
}

\author{
Mauricio Plaza PhD., ${ }^{1, *}$, Oscar Avilés PhD. ${ }^{2}$, William Aperador PhD. ${ }^{3}$ \\ 1,2,3 Department of Mechatronics, Universidad Militar Nueva Granada , Bogotá, Colombia \\ 1,2,3 Mauricio.plaza@unimilitar.edu.co, oscar.aviles@unimilitar.edu.co, William.aperador@unimilitar.edu.co
}

\begin{abstract}
The electronic control technology for mobility and domotics control (home automation systems) can be a great help to people with spinal injuries who have major limitations in the mobility and in the use of devices for normal life activity. The design of different type of technologies to provide to the patient aids is able to increase his quality of life.

A spinal cord injury (SCI) is typically defined as damage or trauma to the spinal cord that in turn results in a loss or impaired function resulting in reduced mobility or feeling.

Typical common causes of damage to the spinal cord are trauma or disease. The resulting damage to the spinal cord is known as a lesion, and the paralysis is known as quadriplegia or quadraplegia / tetraplegia if the injury is in the cervical (neck) region, or as paraplegia if the injury is in the thoracic, lumbar or sacral region.
\end{abstract}

Equipment manufacturers say that designing applications for users with disabilities is not cost-effective. Most of these systems are designed for users who are not disabled; therefore, systems that address disabled users need special interfaces in order to be accessible.

In this paper we present a method for developing an electric and mechanical prototype for quadriplegic people provided that they can perform specific grade of mobility. Using an infrared technique, computer vision technology and mechanical design, users can perform some activities for improve his quality of live and give some grade of independence.

Keywords Electric chair, quadriplegic, spinal injure

\section{Introduction}

\subsection{Complete and Incomplete Spinal Cord Injury}

There are typically two types of lesions associated with a spinal cord injury, these are known as a complete spinal cord injury and an incomplete spinal cord injury. Both of this level can be partial solve whit the design proposed in this research.

A complete type of injury means the person is completely paralyzed below their lesion; this type of injury is most concerned with the research. There are no possibility or therapy and it is necessary a technological solution for the patient mobility. 
Whereas an incomplete injury, means only part of the spinal cord is damaged. A person with an incomplete injury may have sensation below their lesion but no movement, or vice versa. There are many types in incomplete spinal cord injuries, and no two are the same.

Incomplete spinal cord injury can be solving whit the technology used for people with complete paralyses with some simple modifications.



Figure 1. Spinal Cord Injury level

Quadriplegia is caused by damage to the cervical spinal cord segments at levels C1-D8. Damage to the spinal cord is usually secondary to an injury to the spinal vertebrae in the cervical section of the spinal column. The injury to the structure of the spinal cord is known as a lesion and may result in the loss of partial or total function in all four limbs, meaning the arms and the legs. Because the damage produces loss of control in four limbs, the research proposes the use of the jam and head movements to control the mobility and domotic operation (figure 1.)

Typical causes of quadriplegia from damage to the spinal cord are trauma (such as car crash, fall or sports injury), disease (such as transverse myelitis or polio) or congenital disorders, such as muscular dystrophy. It is possible to injure the spinal cord without fracturing the spine, such as when a ruptured disc or bony spur on the vertebra protrudes into the spinal column.

\subsection{Symptoms of Quadriplegia}

Upon visual inspection of a quadriplegic patient, the first symptom of quadriplegia is impairment to the arms and legs.

Quadriplegia is defined in different ways depending on the level of injury to the spinal cord. C1-C4 usually affects arm sensation and movement more so than a C5-C7 injury; however, all quadriplegics have or have had some kind of finger dysfunction. That kind of injure do not permit the control with the limb, but with the only move part like head and jam. 
A person with damage to the spinal cord at the cervical spinal cord segment $\mathrm{C} 1$ (the highest cervical vertebra, at the base of the skull) will probably lose function from the neck down and require permanent assist. In order to provide some degrade of freedom, the design of control technology and special interface are the goal of the research

A person with a C8 spinal cord injury may lose function from the chest down, but still retain use of the arms and much of the hands. In this case the design of technology could be less efficient and with different interface.

The degree of the injury to the cellular structures of the spinal cord is very important. A complete severing of the spinal cord will result in complete loss of function from that spinal segment down. A partial severing or even bruising or swelling of the spinal cord results in varying degrees of mixed function and paralysis.

The prototype proposed is thinking in the high level of quadriplegia. The other type of injure can be solve whit very simple modifications.

\subsection{ASIA impairment scale}

Spinal cord injuries are classified by the American Spinal Injury Association (ASIA) classification. The ASIA scale grades patients based on their functional impairment as a result of the injury, grading a patient from $\mathrm{A}$ to $\mathrm{D}$
A Complete No motor or sensory function is preserved in the sacral segments S4-S5.
B Incomplete
Sensory but not motor function is preserved below the neurological level and includes the sacral segments S4-S5.
Incomplete: motor function is preserved below the neurological level, and more
C Incomplete than half of key muscles below the neurological level have a muscle grade less than 3.
D Incomplete $\begin{aligned} & \text { Incomplete: motor function is preserved below the neurological level, and at least } \\ & \text { half of key muscles below the neurological level have a muscle grade of } 3 \text { or more. }\end{aligned}$
E Normal

\subsection{Spinal Shock Overview}

Spinal shock following a spinal cord injury is a specific term that relates to the loss of all neurological activity below the level of injury. This loss of neurological activity includes loss of motor, sensory, reflex and autonomic function.[7-8].

\subsection{Spinal Cord Injury Levels}


Spinal cord injury levels are used to explain the different severities of paralysis, following an injury to the spinal cord. Generally, the higher the level of injury to the spinal cord, the greater the degree of paralysis will result.

\subsection{Functionality of C1, C2, C3 Quadriplegic}

\subsubsection{Mobility \& Movement}

Limited head and neck movements can be done depending on muscle strength. The research prototype include an oral mouse to solve this problem

\subsubsection{Complete paralysis of arms, body and legs.}

Sympathetic nervous system will be compromised possibility of Autonomic Dysreflexia. Electric wheelchair may possibly be controlled by either neck an oral movement controller; this will vary depending on dexterity. Complete assistance required during mealtimes.

\subsubsection{Personal Care}

Complete personal assistance is required. The person will need assistance with washing, dressing, and assistance with bowel and bladder management. The prototype can free a personal care for mobility and house interface control.

\subsubsection{Domestic Care}

Complete domestic care is required, such as household cleaning, washing of clothes and kitchen duties, preparation of meals and general household duties. Again, the prototype can free a personal care for mobility and house interface control.

\subsubsection{Communication}

A computer may be operated using head movement recognition, mouth stick recognition. Telephone can be used using oral mouse recognition and headset.

\subsubsection{Environmental Control}

The system designed permitting remote control of electronic devices in the immediate surroundings. A person can independently turn lights, radio, and television on and off, answer or initiate phone calls, and unlock a door. Essentially any aspect of the environment can be controlled depending upon the system's complexity.

A hardware and software system, allowing the user programmed or spontaneous control over remote, electrically operated appliances.

The prototype is special technology equipment providing disabled access of the physical 
environment like automatic door openers, environmental control units and modular unit for electric wheel chair control and control units for other assistive technologies [9].

\section{Designing of technology and interfaces for quadriplegic people}

Paralysis is broadly characterized by the loss of muscle control, power and movement to one or more parts of the body. Quadriplegic is a person with damage to the spinal cord at a high level, resulting in sensory and motor function impairment in all four limbs and the trunk. It is caused by damage to the brain or the spinal cord at a high level C1 - C8 - in particular, spinal cord injuries secondary to an injury to the cervical spine. The persons develop a dependency that limits his possibilities and his quality of life. Independence in his daily activities improves notably his autoesteem and liberates his family of an additional responsibility.

Using the knowledge and experience on rehabilitation technology, the research group began thinking of developing new products for people with quadriplegia due to spinal cord injury or other causes. The basic needs are the locomotion, activation of devices as light, television, doors remote control or communications. The present project intends to identify the basic needs of quadriplegic people and the possible solutions using technology of infrared tracking and new design of oral mouse. Diagrams of the Different path result of the jam movements, used for oral mouse design. (Figure 2-5)

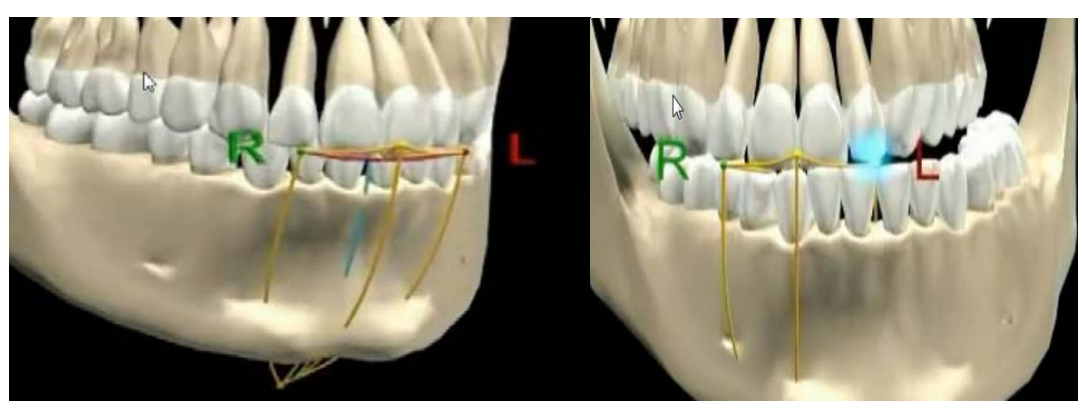

Figure 2. Fontal and lateral movements

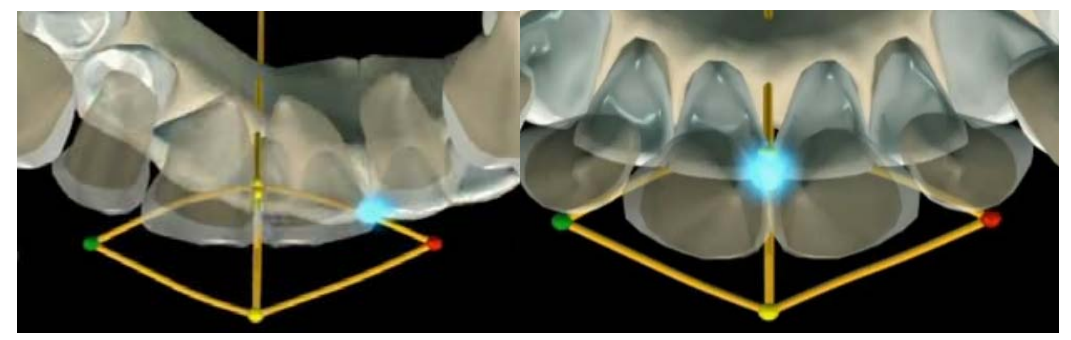

Figure 3. Upper path 


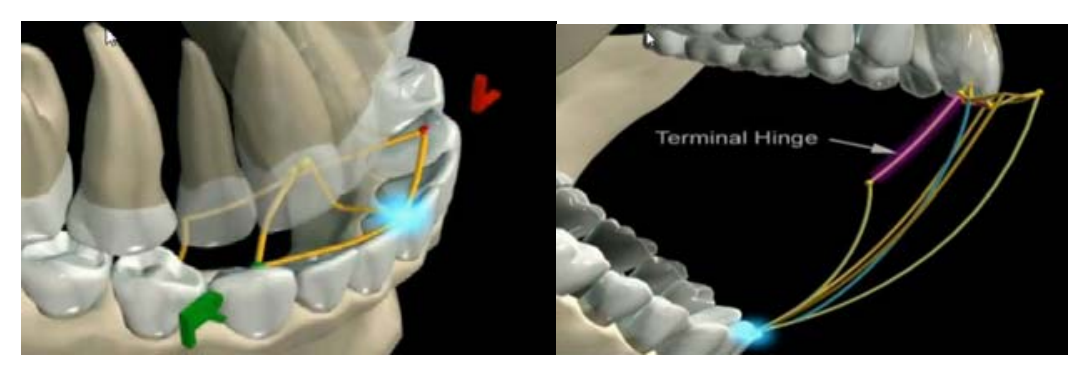

Figure 4. Lowewr path

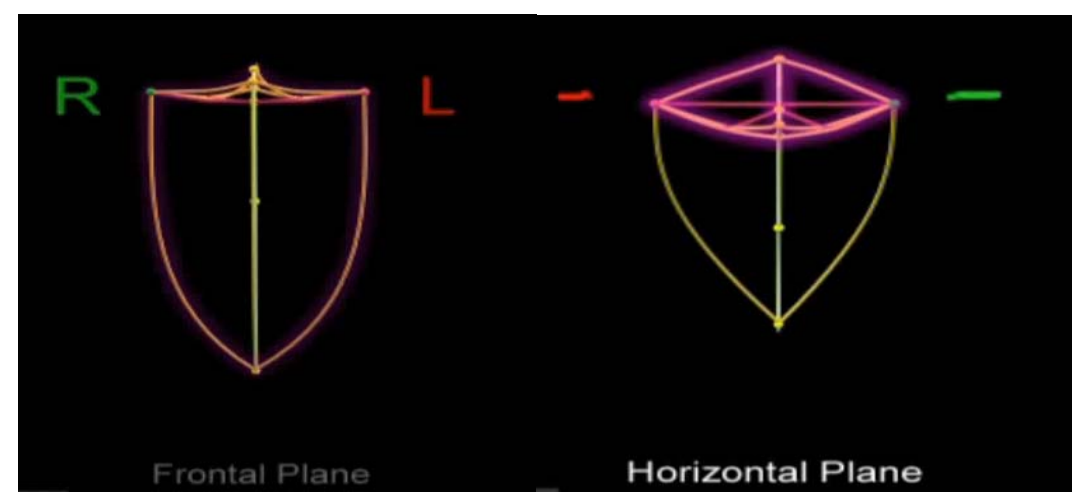

Figure 5. Fontal and horizontal plane path

Quadriplegia is a disorder where an individual loses the use of all four limbs: legs and arms, as well as other parts of their body. In some cases the individual may still have partial movement in one limb or several parts of their body. One of the aims of the project is to provide an opportunity for persons with paraplegia seeking to re-establish themselves in community living. The persons with limitations in his members develop dependency from other people, for his primary needs of movement and common and daily activities. This dependency deteriorates his life quality and of the person dedicated to his care. For this limitation, the autoesteem of the person with limitations is low, creating psychological and physical problems that can be solved partially by means of some type of technology.

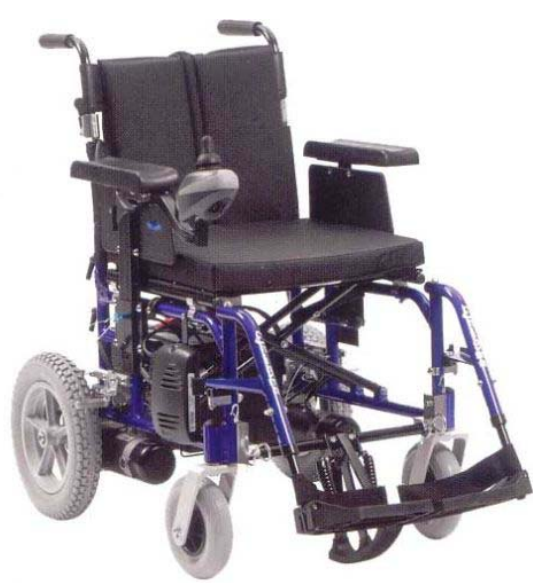

Figure 6. Traditional Electric chair 
Living with and managing quadriplegia is difficult, but not impossible. Those suffering from the condition need to be prepared to make changes in their life and their home. We have an opportunity to help on this process. The devices designed have to take special characteristics like low cost, control with movement of the head, in some cases with finger activation, good accuracy and easily to maneuver. The basic idea of the project consists of develop an infrared tracking device that allows the devices activation by means of the movement of the head to quadriplegic people [1].

In some cases the affected person has the possibility of basic finger movement that allows activating the devices like a switch or the activation by means of a trackball. It will be studied and will leave the use possibility of the two devices depending on the injury type and the mobility possibilities [3]. The development of this device improves the quality of life of the affected people and opens new alternatives for handling different activities in this kind of persons [2]. They hope to use these signals to guide motorized assistance mechanisms that restore mobility in a specific area. The involved costs are low, the operation capacity is simple and his development is possible. The prototype include an infrared tracking system, a computer that processes the signal from the system and a display with the chosen options, a power system for the relay control and a digital system that is the interface with the power unit [4].(figure 7.)

The main objective is to determine the needs of the people with limitations of mobility and to propose solutions with devices bases on infrared tracking and oral mouse.

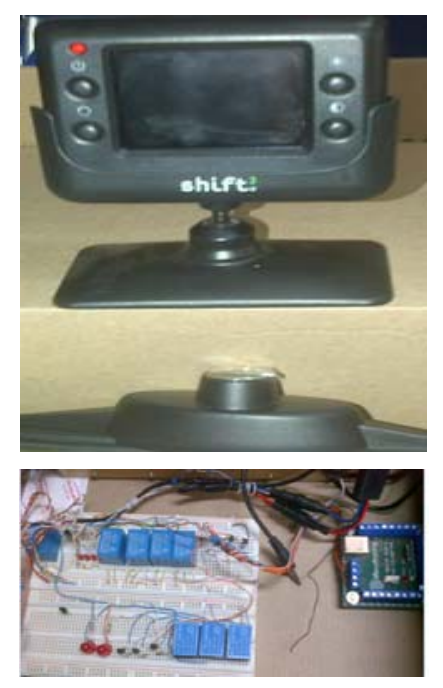

Figure 7. Electronic control prototype

Technology of electric wheelchair control (figure 6.) and domotics systems can really change the life to the people with spinal injuries; especially in the cases of major limitations in the use of the upper limbs. In order to provide an electronic device or system, with the purpose to increase the quality of life and opportunities for participation and inclusion, it is necessary that this process follows a well-established methodology and a multidisciplinary approach (mechanical and electric 
science). Our procedure consists of some phases: 1) Analysis of user needs. At this stage we consider the type of disability 2) Finding aid. We consider the cost, service and facilities, 3) customization of the device. 4) Design of the technology.

The specific objectives are:

- To determine the primary needs of persons with limitations of movement

- To study the possible solutions to obtain a certain percentage of independence

- To use technology of infrared tracking to supply part of the needs of mobility and devices control.

- To escalate the project to different grades of incapability (small movements of hand, total immobility of members...)

- To realize a prototype that allows a person with a certain grade of incapability, to be able to have high level of independence with the control of some devices and mobility in a specific area.

- A person using the final project to control a wheelchair, for example, only has to move your head about going straight ahead or turning left and the chair follows their command. However, they do not have to worry about colliding with obstacles because the wheelchair itself monitors and reacts to its environment.

\section{Methodological Approach}

- Bibliographical review. Allows knowing the current advances in the devices control for quadriplegic systems. It allows the comparison of alternative systems as control with EEG. It allows the review of current technologies in infrared systems.

- Surveys in the health sector. It allows understanding the specific needs in persons with these limitations. It allows validating the proposal and to determine if there are improves in the model.

- Prototype preparation and simulation.

- Prototype test with quadriplegic people and improvement of the model.

- Final prototype development

- Technical test and prototype improvement, we have done is combine the intelligence of the person with the artificial intelligence of the device.

- Field test

- Model improvement

- Final prototype development

\section{Conclusion}

The final prototype improves the quality of life of quadriplegic people. The prototype must have the possibility of allowing the control of electrical wheel chairs, of some devices connected to a wireless control system, only by means of the movement of the head. 
We hope that the solution should be practical, easy use and should not generate very high costs, as well as safety and immunity to the noise. These characteristics allow that the system should be robust characteristics and easy to reproduce. The persons who have this system must have the possibility of easy movement in a specific area by means of the movement of some part of the body that still has control.

The system has the capacity to adjust itself to the characteristics of each individual driver, and thereby is able to improve the efficiency with which it senses the driver's commands.

People, for example, do not consciously send commands to every muscle in each leg in order to walk and do not think where to step to avoid an. Similarly, a wheelchair-bound user of the project simply has to send the signal to go in a certain direction and the chair figures out how to get there.

A person using the final project to control a wheelchair, for example, only has to move your head about going straight ahead or turning left and the chair follows their command. However, they do not have to worry about colliding with obstacles because the wheelchair itself monitors and reacts to its environment.[5]

The fact that is an infrared system allows being immune to electrical noise, as generated by the electric chairs and allows low costs and easy design of engineering. These characteristics are desirable to reduce costs and to make the project possible.

\section{References}

[1] A. Biocca, A survey of position trackers, Presence: Teleoperators and Virtual Environments, v.1 n.2, p.173-200, Spring 1999

[2] A. B. Zoss, H. Kazerooni, A. Chu, "Biomechanical design of the Berkeley Lower Extremity Exoskeleton (BLEEX)“, IEEE/ASME Trans. Mechatronics, vol. 11, no. 2, pp. 128-138, 2006.

[3] Adam C. Esser, MD, James G. Koshy, Ph.D, Henry W. Randle, MD, Ph.D. "Ergonomics in Office-Based Surgery: A Survey-Guided Observational Study”. American Society for Dermatologic Surgery, Inc. November 2007

[4] A. J van den Bogert, "Exotendons for assistance of human locomotion”, Biomed. Eng., Online, 2:17, 2003.

[5] A. M. Dollar, H. Herr, "Lower Extremity Exoskeletons and Active Orthoses: Challenges and State-of-the-Art”, IEEE Trans. Rob., vol. 24, no. 1, 2008.

[6] Axel Mulder. "Human movement tracking technology". Technical report, School of Kinesiology, Simon Fraser University, 2000

[7] Kenneth Meyer , Hugh L. Applewhite , Frank

[8] Olivier Faugeras, Three-dimensional computer vision: a geometric viewpoint, MIT Press, Cambridge, MA, 2001

[9] R. T. Tsai "An Efficient and Accurate Camera Calibration Technique for 3-D Machine Vision", Proceedings of the IEEE Conference on Computer Vision and Pattern Recognition, 2001. 